\title{
The Textile Machineries Exhibited in the International Trade Fair Held in Tokyo in 1961
}

\footnotetext{
The 4th international trade fair was held at Harumi pier near the center of Tokyo from 17th April to 7th May of this year.

Twenty eight countries participated in the fair and not only the scale of the fair was big but the number of visitors increased from 2.5 million to 1.9 million in comparison with the last year.

Most of the textile machines were exhibited in hall No. 10 . In comparison with the last year's Osaka international trade fair, the number of exhibitors and exhibits were less in number, but there was progress in the content of the exhibits.

Here we are giving a report on it.
}

By S. Ishikawa, M. Yamaguchi, T. Sakai, S. Tabata, T. Iizima, F. Bekku, N. Oguchi,

T. Kawai, J. Shimizu and G. K. Joarder, Members, TMSJ

Tokyo Institute of Technology, Meguro-ku, Tokyo

1. Spinning Machine

(1) D-type card Ohtori Kiko., Ltd.

(2) Auto even frame OM Lid. Auto even draw frame Auto even draw head

(3) Narrow spinning machine Howa Machinery Ltd.

(4) PADS high drafting spinning machine Fukushima Ltd.

(5) HA-type insert Nihon Seiko Co., Lid.

2. Weaving Preparatory Machine

(6) Kamitsu's SP high-speed precision winder Kamitsu Setsakusho Co., Ltd.

(7) Murata's $\# 52$ super-speed precision winder Murata Textile Machine Co., Ltd.

(8) Kamitsu's fully automatic super high-speed winder Kamitsu Seisakusho Co, Ltd.

3. Weaving Machine

(9) Howa KZ-100 type automatic loom Howa Machinery Co., Ltd.

(10) Sakamoto KS-A type automatic loom Enshu Ltd.

(11) Hirano's HGR type $4 \times 1$ automatic loom Hirano Loom Works Co., Ltd.

(12) Toyoda GB II automatic loom Toyoda Automatic Loom Works Ltd.,

(13) Tsudakoma's LMD type $4 \times 1$ automatic loom Tsudakoma Industrial Co, Ltd.

(14) Hokuriku's JS type $4 \times 1$ automatic loom Hokuriku Kikaikogyo Co., Ltd.

(15) Hiraiwa's clompton and norse PAPA $4 \times 3$ automatic loom Hiraiwa Iron Works Co., Ltd.

(16) Murata-maxbo air jet loom Murata Textile Machine Co., Ltd.

\section{Knitting Machine}

(17) T-HWM type single tricot machine Tsugami Seisakusho Co., Ltd.

(18) CWE ribbed hose sock knitter Daito Saiki Co., Ltd.

\section{Dyeing Machine}

(19) Self rotating spraying tube type automatic hank dyeing machine Toyo Kakoki Works Ltd. High-temperature and high-pressure spraying automatic dyeing machine Suzuki Seisakusho Co., Ltd.

(20) High-temperature and high-pressure hank dyeing machine Suzuki Seisakusho Co., Ltd.

(21) High-pressure jigger Kyoto Machinery Co., Ltd.

(22) High-pressure jigger for universal samples Nippon Dyeing Machine Mfg., Co., Ltd. High-temperature and high-pressure dyeing testing machine Nippon Dyeing Machine Mfg., Co., Ltd. 12 color, high-pressure and high-temperature dyeing machine Nippon Dyeing Machine Mfg., Co., Ltd. 
6. Finishing Machine

(23) Fully automatic centrifugal separator Tanabe Iron Works Co., Ltd.

(24) Vertically suspended type directly centrifugal separator Tanaka Machine Co., Ltd.

(25) Ultra filter Miura Kagaku Sochi Co., Ltd.

(26) Endless cross belt filting machine Daiki Rubber Industry Co., Ltd.

(27) IDI type small tension taking-up machine Ichikin Kogyosha Co., Ltd.

(28) Ebara-inflico cyculator Ebara Seisakusho Ltd.

7. Sewing and Other Finishing Machine

(29) Tsugami kilting machine Tsugami Seisakusho Co., Ltd.

\section{Textile Testing Machine}

(30) Micro-scope and Projector Hitachi Ltd., Asahi Sokki Co., Nihon Electric Co., Union Optical Co.

(31) Thickness measuring meter by $\beta$ ray (WD.626 B typo), Iwasaki Tsushin Co., Ltd.

(32) Durability testing machine Toyo Rika Kogyo Co., Ltd.

(33) Dew point hygrometer Sumitomo Electric Industry Co., Ltd.

(34) Tensile testing machine Toyo Seiki Seisakusho Co., Ltd.

(35) Color Difference Meter Nihon Color Machine Co., Ltd.

(36) Calender gauge Densoku Kogyo Co., Ltd.

(37) Humidity measuring instrument for rotating roller Shinko Eleciric Instrument Co., Ltd.

\section{Spinning Machine}

The number of the exhibited machines were few in number in comparison with the usual years. Although there were no specially new things, improvements in some parts could be seen.

\section{(1) D-type Card (Ohtori Kiko exhibited only)}

This machine was exhibited in two previous years continiously. A second taker-in roller has been set between the first taker-in roller and the cylinder as shown in Fig. 1. The efficiency of separating dirts is high due to the use of air flow and mote knife. This was so long exhibited cooperatively with Daiwa Spinning Co., Osaka Kiko Ltd,, and Otori Kiko Ltd.

This machine helps to increase production and its degree of opening power is high. Therefore, ordinary cards can be replaced by this machine.

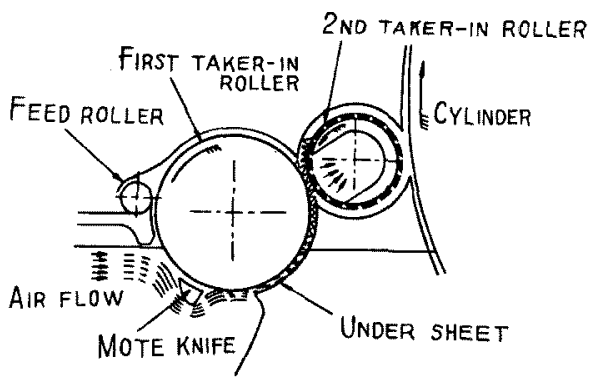

Fig. 1 Main part of D-type Card

\section{(2) Auto Even Draw Frame (O-M Ltd.) Auto Even Draw Head (O-M Ltd.)}

These machines were designed for high productivity and automatizing. There are $\mathrm{FCL}$ type and DCL type and both of them are same in mechanism. The main differing part of this machine is that the front roller of the previous one is constant where as, the back roller of the later is constant.

Fig. 2 shows the outlook of this machine. Each delivery head of it is equipped with seperate automatic controller for the sliver. The production can be done at a speed of $120 \sim 150 \mathrm{rpm}$. The doffing of sliver cans and carrying it to the next process is done automatically.

The cards can be joined by convair belts and a automatic and balanced supply is possible.

FCL type is shown in Fig. 3 and the small movement of the bottom measuring roller due to the irregularity of each sliver is amplified and summed up by the lever mechanism. This signal is am. plified by link mechanism and hydraulic servo motor, which moves the belt shiftor of the cone pulley. The main drafting ratio 4.5 to 5.5 is done between 1st and 2nd roller. The third roller rotates at a constant speed and a control of $\pm 18 \%$ against the draft ratio of 1.5 can be done between this roller and the measuring roller. A tension drafting at a ratio of 1.5 is given between the third and the second roller and the strand is widened by the expander to make the main drafting easier.

When the can is filled to the fixed amount which is set at the auto-counter, the machine stops and the filled can is replaced by the open can automati- 
cally. The shifting of open and filled can is done by convair belts.

The outlook of this type is shown in Fig. 4 and the mechanism is shown in Fig. 5.

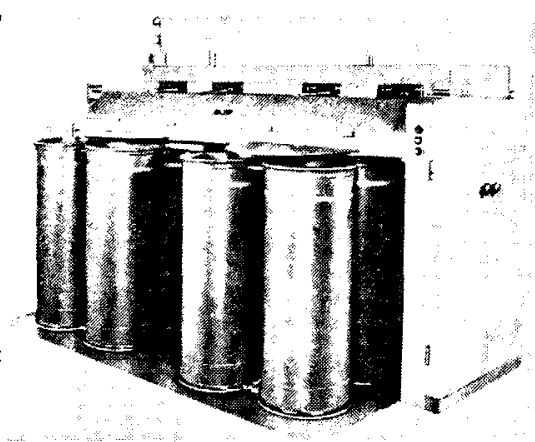

Fig. 2 Auto even draw frame

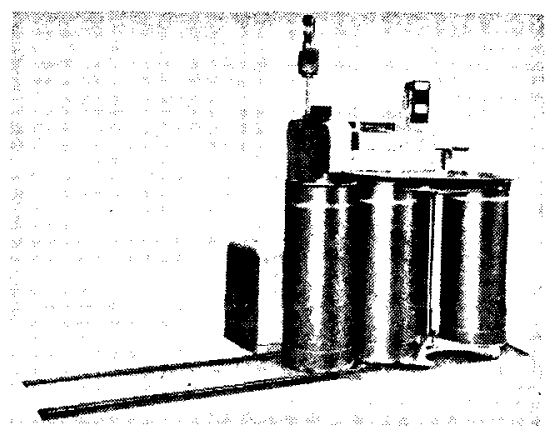

Fig. 4 Auto even draw head

\section{(3) Narrow Spinning Machine (Howa Machinery Co., Ltd.)}

The installation area of this machine is small and it is attached with high drafting equipment of $\mathrm{NH}$ SN type cradle with double aprons. The angle of the roller is $65^{\circ} \quad$ The rotation is smooth and can be speeded highly due to the use of roller bearing. The outlook of this machine is shown in Fig. 6.

The width of this machine is $610 \mathrm{~mm}$ (24 in.) and both sides of it can be used for spinning. The distances between spindles are according to the diameter (44, 50, and $56 \mathrm{~mm}$ ) and are $69,85,76.2 .88 .9$ $\mathrm{mm}$ respectively. The lift is $178 \sim 280 \mathrm{~mm}$. The

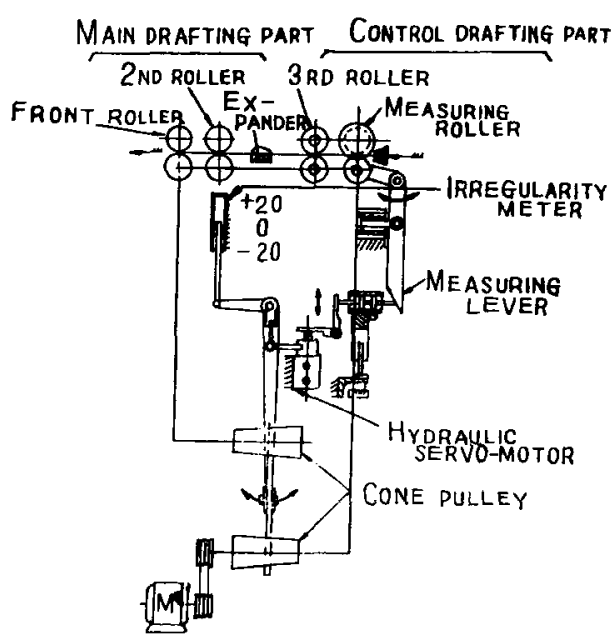

Fig. 3 Control system of Auto even draw frame

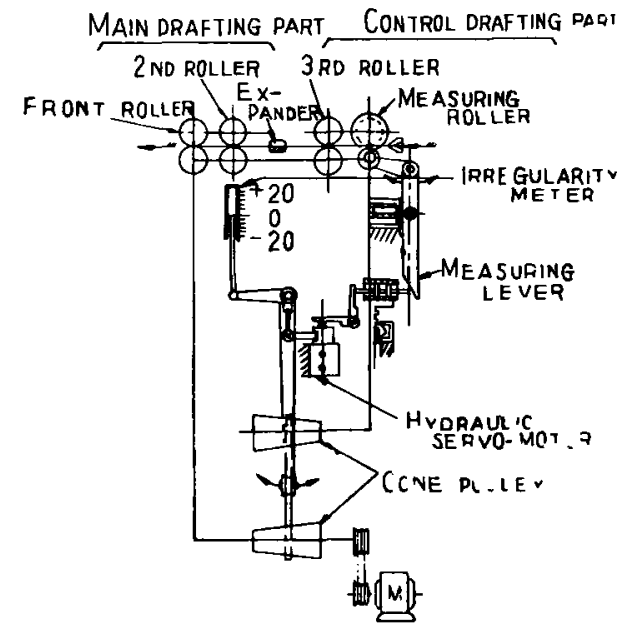

Fig. 5 Control system of Auto even draw head

yarn leaving the front nip comes just at the top of the spindle shaft as has been shown in Fig. 6 . This helps a good spinning. This company's own UDIV type top roller has been used and all types of cradles can be used besides NH-SN type. Moreover, the anti-ballooning equipment works separately and through the whole lifting position the tension of the yarn is almost equal.

Tin pulley has been used instead of tin roller and the ring rail is automatically lowered when the bobbin is full. 


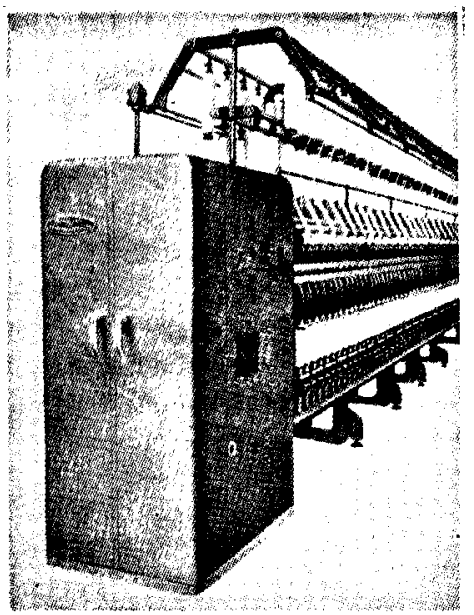

Fig. 6 Howa's narrow spinning machine

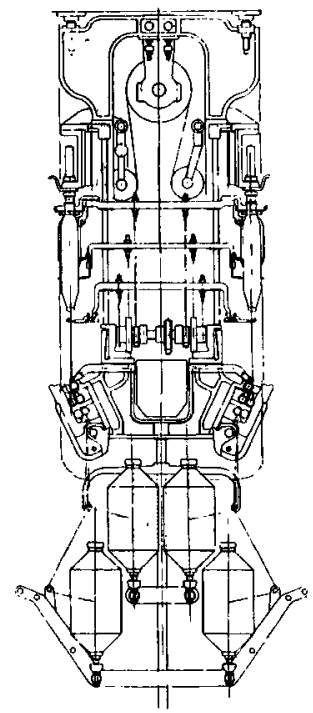

Fig 7 The cross-section of Howa's narrow spinning machine

\section{(4) PADS type High Drafting Spinning Machine}

The super high drafting spinning machine is designed for fibers of long staples. Many difficult problems have been solved for high drafting long fibers. As a result, it can draft at the ratio of $76.7 \sim 150$ in a simple manner and if a special kind of equipment is attached, it can draft at the ratio of 250. In case of man-made fiber, a rotation of 15000 is possible for the spindle. There are many electric controlling devices such as stop motion for full bob- bin etc. and the speed can be changed by variable pitches.

The maximum allowable length of the fiber is $175 \mathrm{~mm}$, the lift is 9 in, the rotation of the spindle is $6200 \sim 10000$, the draft ratio is $200 \sim 980 \mathrm{t} / \mathrm{m}$, and the width of ring is $50 \mathrm{~mm}$.

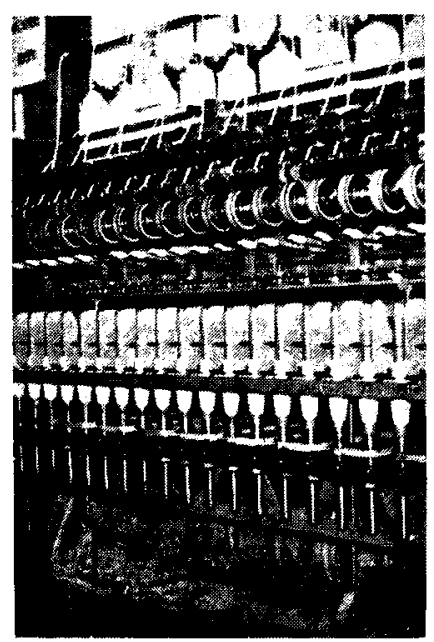

Fig. 8 PADS high drafting spinning machine

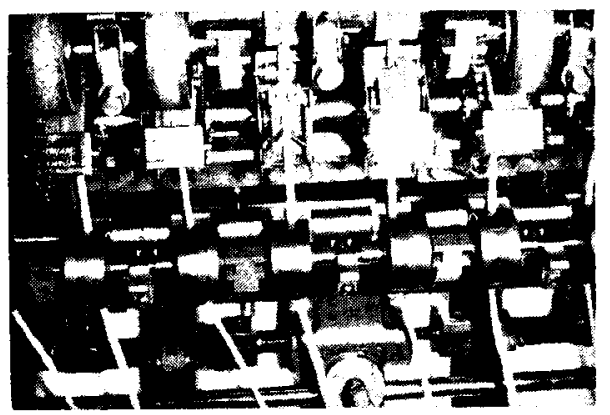

Fig. 9 Drafting part of PADS spinning machine

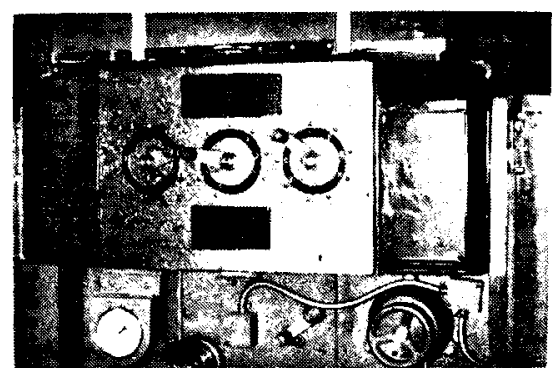

Fig. 10 Electric Controlling Device 


\section{(5) HA-type Insert (Nihon Seiko Co.)}

This company has long experience in making bearing and HA-type Insert has been made by this company's own design. It has three advantages namely, good vibration absorption mechanism,

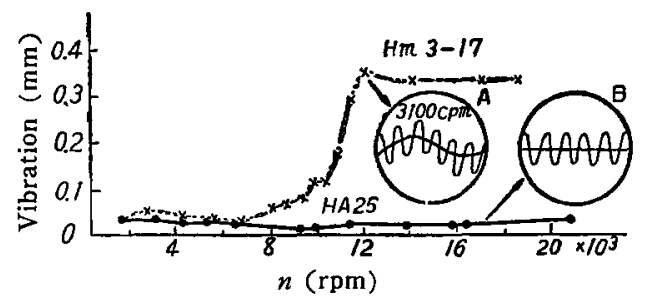

full bobbin

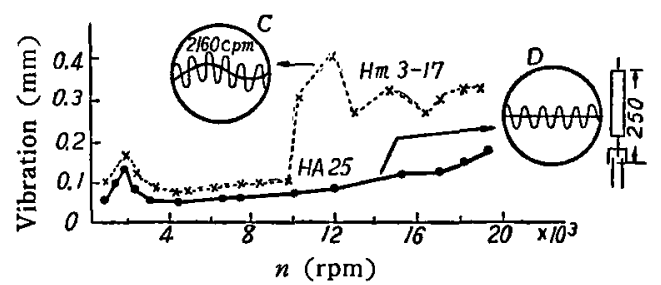

Fig. 11 simpleness in maintenance and decrease of power consumption. Fig. 11 shows its vibration charac. teristic. They are as follows.

1) Primary vibration is small.

2) The peak of the secondary vibration shifts to high speed.

3) The limit of use between the primary and secondary vibration is wide. Therefore it has no self vibration up to $20,000 \mathrm{rpm}$. The consumption of power has been clearly explained in Fig. 12.

Consumption of power by one spinning machine (384 spindles)

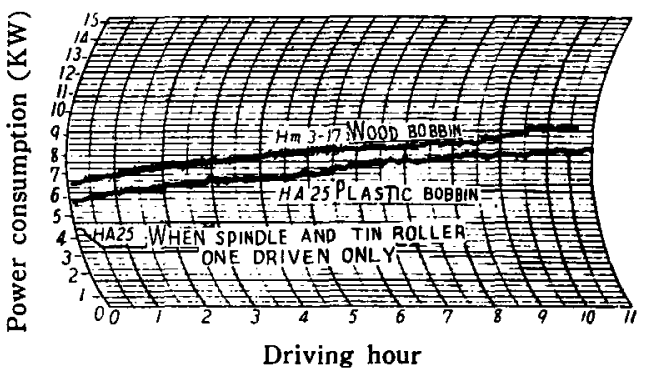

Fig. 12 Comparison of power consumption

\section{Weaving Preparatory Machine}

The exhibited warp winders were D-type High Speed Winder by Ishikawa Seisaku sho Ltd., High

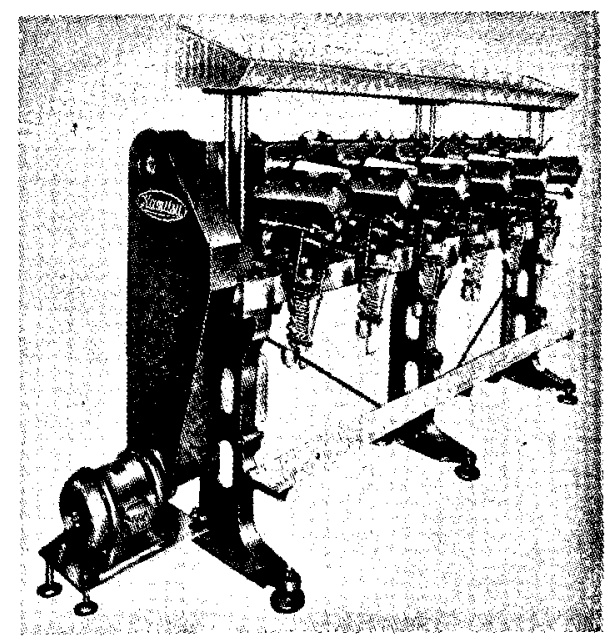

Fig. 13 Kamitsu's SP-type high speed precision winder
Speed RT Winder with Slub Catcher and SP Type Precision High Speed Winder by Kamitsu Seisakusho Ltd., Abott Type $\# 10$ Fully Automatic RT Winder, \#52 Super High Speed Precision Winder by Murata Textile Machinery Co., Ltd. The exhibited weft winders were Abott-type Radial Fully Automatic Quller by Ishikawa Seisakusho Ltd. Fully Automatic High Speed Pirn Winder by Kamitsu Seisakusho Ltd., Koa Super Self Pirn Winder by Koa Spinning Ma. chine Co. etc. There was a TS 101-type Fully Automatic Bobbin Winder for sewing thread by Torii Iron Works Ltd.

(6) Kamitsu's SP type High Speed Precision Winder

(7) Murata's \#52 Super High Speed Precision Winder (Murata Textile Machinery Co., Ltd.) Murata's \#52 Super High Speed Precision Winder is same as Kamitsu's SP Precision Winder (exhibited in last year) 
These are designed for filament yarns of acetate, viscose and synthetic fibers. The rotation of the spindle can be raised to $2000 \sim 2500 \mathrm{rpm}$ (winding number: 2.5) and it is possible to have the yarn velocity of $700 \sim 800 \mathrm{~m} / \mathrm{min}$. The traversing motion of the yarn guide is given by the skrolled cam. The yarn guide does not touch the winding body directly and a suitable pressure is given by the rotating roller of roller bell type. There arises many problems with the increase of yarn layer as the rotation of the spindle is two times greater than the ordinary ones. The traversing part of Kamitsu's SP type winder changes its position with the increase of the yarn layer where as the position of the spindle is constant. In Murata's $\$ 50$ type winder, the traversing part is fixed where as the position of the spindle is changed. The decrease of spindle rotation is done as shown in Fig. 15. The tension control device is of Gate type.

\section{(8) Kamitsu's Super High Speed Fully Automatic Pirn Winder (Kamitsu Seisakusho Co., Ltd.)}

Kiddi compensator having feed back circuit was used so long. Kamitsu's pirn winder also has the tension device with the feed back circuit. The mechanism is shown in Fig. 16. The position of the lever changes with the variation of tension by the change of magnetic force acting on the disk and the tension is regulated. The tension in Kiddi type is regulated from the change of the pressure of the drawing yarn which changes the space between the disks, where as in Kamitsu's type the pressure is given directly on the disk. Generally cotton wastes accumulates largely in the space between the disks and affects the motion where as in Kamitsu's type these are always cleaned by air flow.

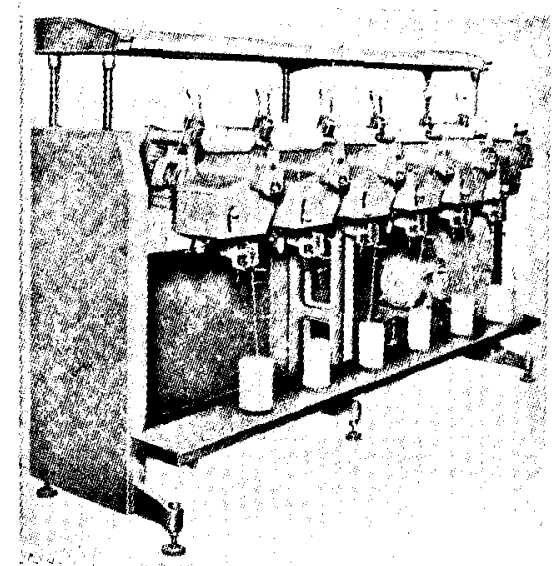

Fig. $14 \$ 52$ Ultra high speed precision winder

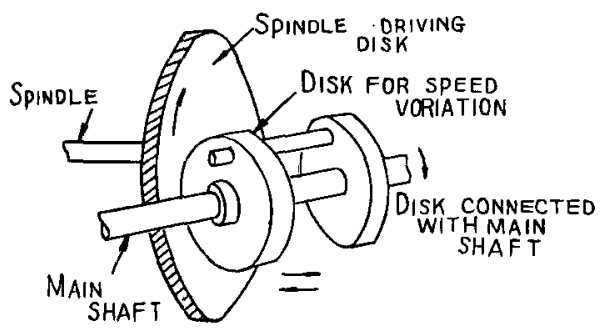

Fig. 15 System of speed variation ( $\$ 52$, SP-type)

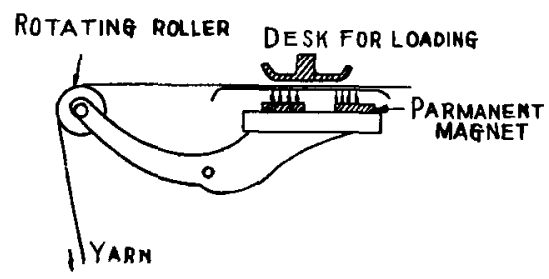

Fig. 16 Tension device

\section{Weaving Machine}

The number of the exhibited looms in this year is less in comparison with the last year and 9 com. panies showed their typical looms. Chechoslovakia's water jet loom was shown for the first time and with the air jet loom it was an attraction to the fair. Some of the looms were same as shown in the previ. ous year and others were high-speeded and modernized in design. Various kinds of healds and its accessories were exhibited by Maruyama Seisakusho Ltd.
(9) Howa KZ-100 Automatic Loom (Howa Ma-
chine Co., Ltd.)

This is the same one as shown in the last year which is a high-speed bobbin changing automatic loom without any top rail. The box magazine has the maximum capacity of 240 spools and comes to replenishing position by its own weight. The end of the weft yarn in the spool is held by the brush attached on the moving belt. The standard revolution for a reed width of $44 \sim 58 \mathrm{in}$. is $190 \sim 210 \mathrm{rpm}$. It can weave from light cloths to berbery and No. 8 canvas cloths. The broad cloth of 60 count was 
being woven in the fair by a loom of reed width $50 \mathrm{in}$. at a speed of $230 \mathrm{rpm}$. the semi-positive let-off mechanism has been applied.

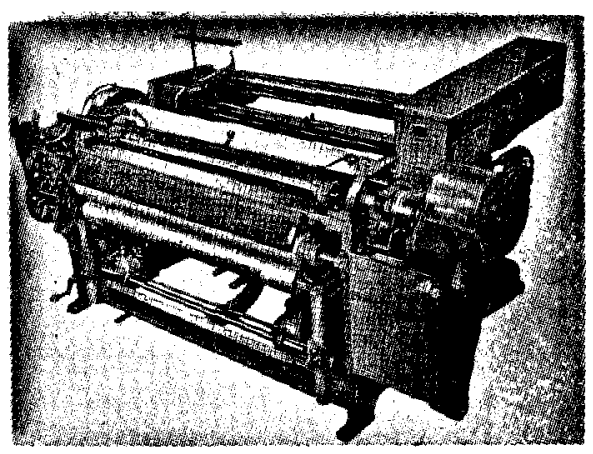

Fig. 17 Howa KZ-100 type automatic loom

\section{(10) Sakamoto's KS·A-type Automatic Loom}

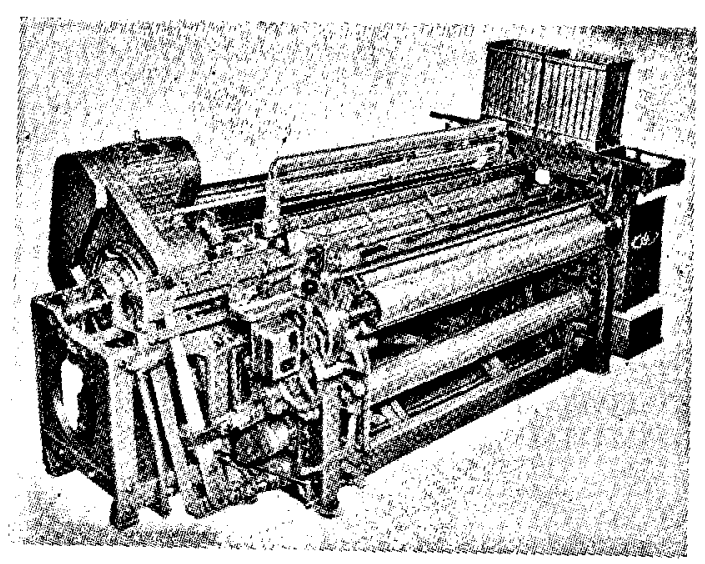

Fig. 18 Sakamoto's KS-A type automatic loom

It is the same as shown in the previous year which is a high-speed, bobbin changing, and non-stop automatic loom. The spools can be kept in two magazine boxes having a capacity of 96 each. When one gets empty, it is replenished by another automatically. The weft end at the replenishing position is held by air sucktion. As a result, the shock due to the replenishment of the spool is less and there by the breaking of the end is lessened. The central picking motion is given by the fixed picker and a mechanism for linear motion of link type is being used instead of shoe. Air bumper and air shuttle stopper have been used. An air pressure of 0.1 $\mathrm{kg} / \mathrm{cm}^{2}$ is blown to the shuttle stopper, which helps to make the shuttle stopper small and light and it prevents any accumulation of dirt. Moreover the oil-damper is used to relax the impact force on the first reed when the first reed stops suddenly.

\section{(11) Hirano's HGR-type $4 \times 1$ Automatic Loom (Hirano Loom Works Co., Ltd.)}

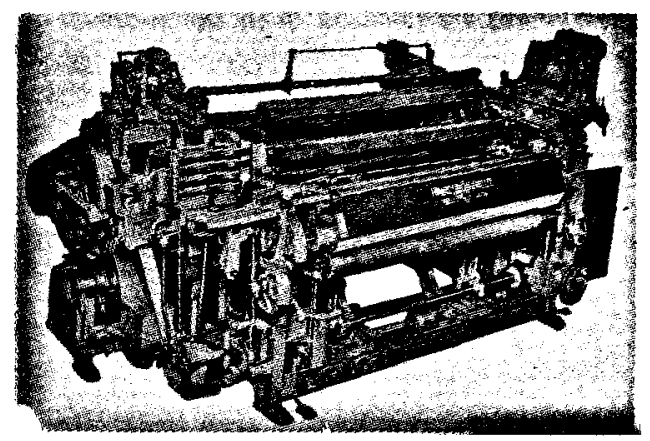

Fig. 19 Hirano's HGR type $4 \times 1$ automatic loom

It is a bobbin changing automatic loom without top lace and having 4 boxes at one side. The width of the reed is 50 inches, and it has positive warp let-off motion. Gingham cloth by Tetoron and cotton blended yarn was being woven in the fair.

(12) Toyoda's GB II-type Automatic Loom (Toyoda Automatic Loom Co.)

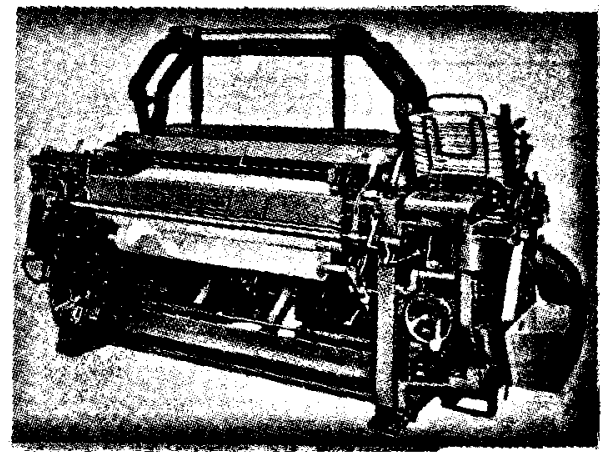

Fig. 20 Toyoda's GB II type automatic loom 
It is an improved type of that shown in the previous year. It is a high-speed shuttle changing automatic loom for thick cloth and is suitable for light canvas and other industrial cloths. The diameter of the yarn beam is $660 \mathrm{~mm}$ and the maximum diameter of the cloth beam is $445 \mathrm{~mm}$. The center of the cloth beam moves along an arc while the diameter of the beam increases. Oil damper is used to prevent impact force on the sley and the central picking motion is done. Moreover, as one of the gears in the let-off mechanism is a change wheel, the density of the cloth can be varied widely.

\section{(13) Tsudakoma's LMD-type $4 \times 1$ Automatic Loom (Tsudakoma Industrial Works Co. Ltd.)}

It is the same shuttle changing automatic loom without top rail as shown in the previous year. The exhibited loom has a reed width of $125 \mathrm{~cm}$ and is driven by push-button. It was weaving 4 coloured cloth by $40 \mathrm{~d}$ Tetoron filament yarn in warp and $30 \mathrm{~S}$ Tetoron yarn in weft at a speed between $175 \sim 180$ rpm. The weft feeler is of photo-electric device and the droppers in electric system are placed in 6 series. A special gauge is fixed in front of the cloth fell to fix its position.

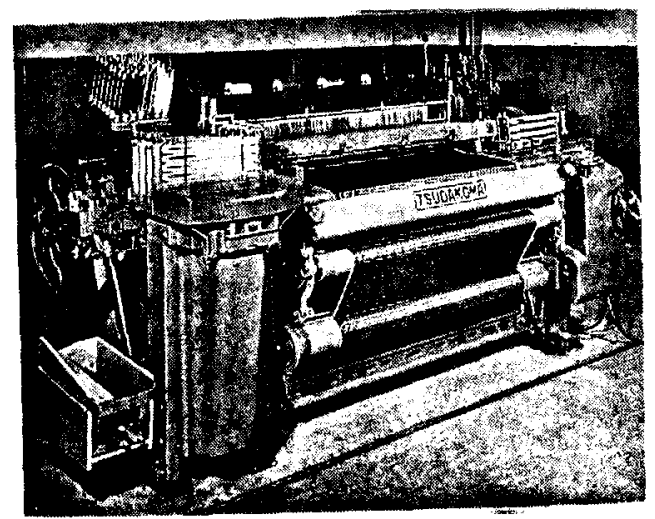

Fig. 21 Tsudakoma's LMD type $4 \times 1$ automatic loom

\section{(14) Hokuriku's JS-type $4 \times 1$ Automatic Loom (Hokuriku Machinery Kikaikogyo Co., Ltd.)}

It is a shuttle changing automatic loom without top rail. The exhibited loom has a reed width of $151 \mathrm{~cm}$. The starting and controlling of motion is done by the magnetic clutch and brake, and the operation of the motion is done by both push button and handle. Moreover, the regulation of velocity is done by velocity regulator which is set on the operation table, and the weft feeler is of photo-electric device. The oiling is done by pump.

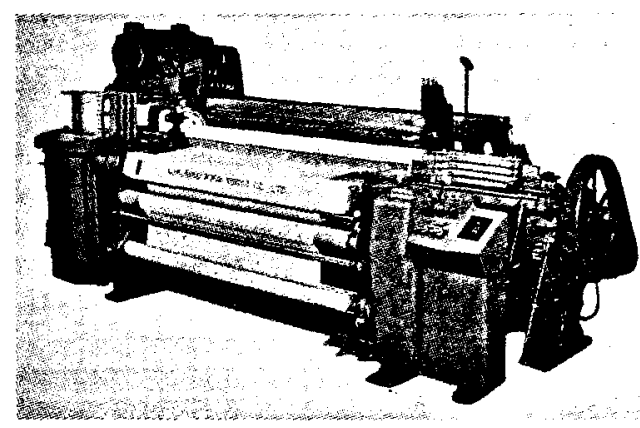

Fig. 22 Hokuriku's JS type $4 \times 1$ automatic loom

\section{(15) Hiraiwa's Clompton and Norse PAPA-type $4 \times 3$ Automatic Loom (Hiraiwa Iron Works Co., Ltd.)}

This loom has been manufactured by the technical cooperation with Clompton \& Norse Co. This is $4 \times 3$ bobbin changing automatic loom for worsted and woolen cloths.

The warp stop motion is done electrically, and the weft-feeler is of photo-electric type. The taking-up is continious, and the end of the weft is dragged in an accumulator by air sucktion, which prevents end breakage due to the shock caused by the replenishment of the shuttle.

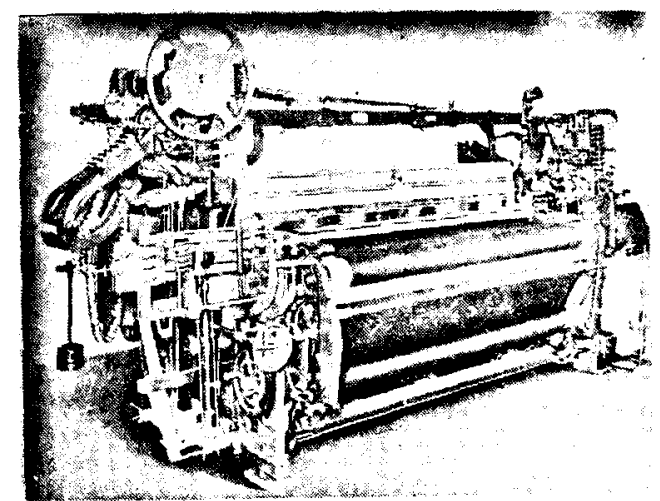

Fig. 23 Hiraiwa's clompton and norse PAPA-type $4 \times 3$ automatic loom 


\section{(16) Murata's Maxbo Air Jet Loom (Murata Textile Machine Co., Ltd.)}

It was weaving a cotton gabardin by $30^{\prime} \mathrm{S}$ double yarn in the fair. The warp density of the cloth was 69 ends/in and the weft density was 65 picks/in. The reed width is $1 \mathrm{~m}$ and 8 healds can be used together. The revolution is $320 \sim 400 \mathrm{rpm}$ and the noise is very little due to non-picking motion. The power required is $2.5 \mathrm{HP}$ and its self weight is $1,400 \mathrm{~kg}$. It has a positive easing motion and the warp tension is controlled by the tension back roller.

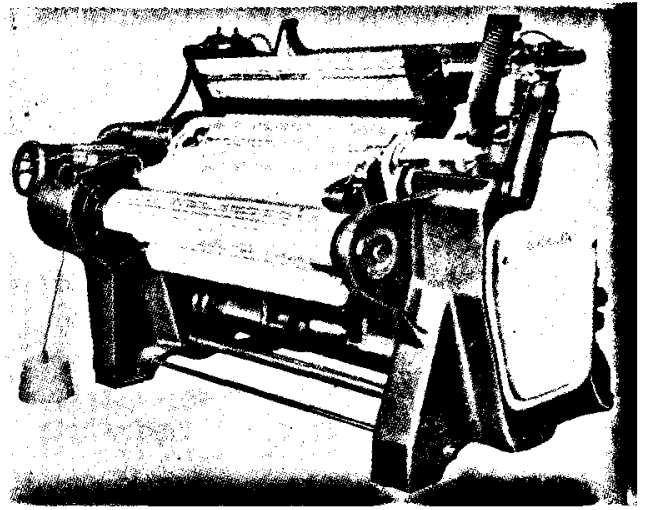

Fig. 24 Murata's maxbo air jet loom

\section{Knitting Machine}

High-speed T-HWM type was the only warp knitting machine by Tsugami Co., shown in the fair. Nagata Seiki's R-LJ 3 Links Jacquard Sock Knitter, R-JJ Top Jacquard Sock Knitter, C-I-C Colour in Colour Sock Knitter, TS Sock Knitte, K-2 FS-Z Micro Mesh Nylon Stocking Knitter, Daito Seiki's CJLT Top Jacquard Links Stocking Knitter, CWE Ribbed Hose Stocking Knitter, etc., were the exhibited circu. lar knitting machines. Most of these machines were shown in the previous year.

(17) T-HWM Single Tricot Machine (Tsugami Seisakusho Co., Ltd.)

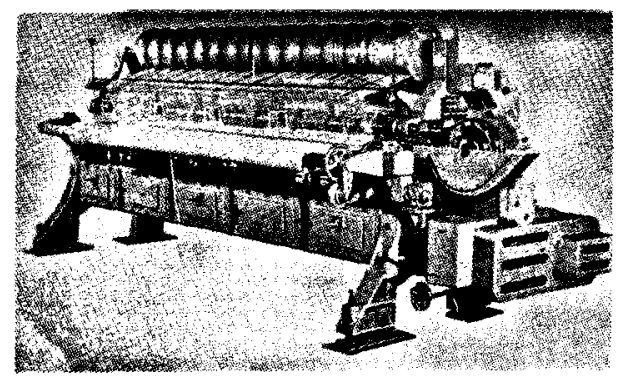

Fig. 25 High-speed warp knitting machine
Magnesium alloy has been used for the reed of this machine instead of aluminium alloy for the purpose of making it light. Moreover, the moving limit of the reed, needle, and sinker has been made small, and a revolution of $900 \mathrm{rpm}$ is possible in the place of $800 \mathrm{rpm}$.

\section{CWE Ribbed Hose Stocking Knitter (Daito Seiki Co., Ltd.)}

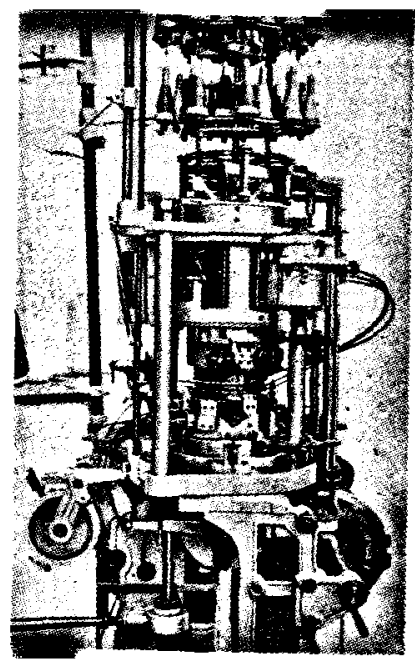

Fig. 26 CWE ribbed hose stocking knitter

\section{Dyeing Machine}

All the exhibited dyeing machines were of hightemperature and high-pressure type. This system has been applied from 2 or 3 years before for dyeing hydrophobic fibers.

(19) Self Rotating Spray Tube Type Antomatic Hank Dyeing Machine TKS-11-Z 2-type (Toyo 
Kakoki Works Co. Ltd.)

High-Temperature and High-Pressure Spraying Automatic Hank Dyeing Machine (Suzuki Seisakusho Co., Ltd.)

The dyeing fluid is sprayed through the holes of the pipe and the hank is rotated around it. Hank yarns weighing 6 9 $\mathrm{kg}$ can be hanged on one pipe (spraying tube). Improvement has been done in the rotation and supporting of the end of the pipe to prevent entwining of the yarn. A sampling instrument for small hanks has been attached in the dyeing machine by Toyo Chemical Machine Manufacturing Co. The maximum capacity is $50 \mathrm{~kg}$ in Suzuki's dyeing machine. The highest dyeing temperature is $138^{\circ} \mathrm{C}$ and the highest pressure of $3 \mathrm{~kg} / \mathrm{cm}^{2}$ is not different to ordinary ones.

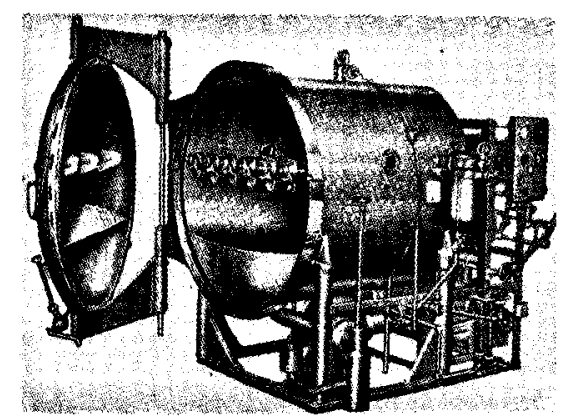

Fig. 27 High-temperature and high-pressure spraying automatic hank dyeing machine

(20) High-Temperature and High-Pressure Hank Dyeing Machine (Suzuki Seisakusho Co., Ltd.)

This is of package of system and other parts are same as above. The propeller for giving up and down flow to the dyeing fluid is attached in the out side of the cylinder, which makes the life of the metal bearing longer. Fleece, top, and cheese dyeing can be done by changing carrier.

\section{Finishing Machine}

There were some machines attached with auto. matic controller and modern chemical equipments.

\section{(23) Fully Automatic Centrifugal Seperator (Ta- nabe Iron Works Ltd.)}

The functions of starting, oil supplying washing, dehydrating, controlling, velocity changing, scrapping

\section{(2I) High-Pressure Jigger (Kyoto Machinery Co.,} Ltd.)

The use of sampling instrument of burning type has been discarded and a pressure resistant glass window and lightening arrangement has been attached instead.

(22) High-Pressure Universal Sample Jigger (Nihon Dyeing Machine Mfg., Co., Ltd.)

High-Temperature and High-Pressure Dyeing Testing Machine (Nihon Dyeing Machine Mfg. Co., Ltd.)

12-color, High-Temperatures and High-Pressure Dyeing Machine (Nihon Dyeing Machine Co.)

The effective width is $254 \mathrm{~mm}$, and the maximum winding diameter is $80 \mathrm{~mm}$.

12-color, high-temperature and high-pressure dyeing testing machine has 12 pots for testing various colors at one time. Dyeing in 12 different colors can be done at one time and fleece, yarn and cotton can be dyed by changing the carrier.

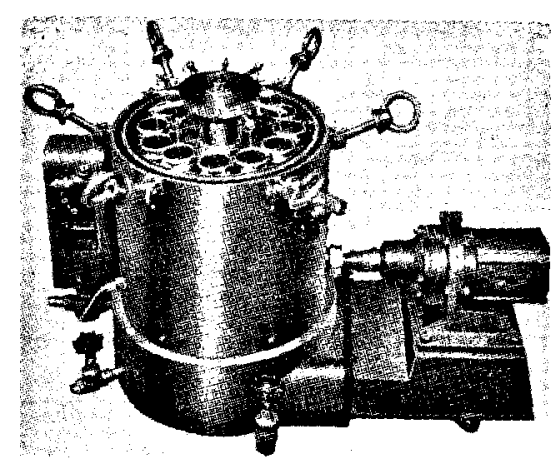

Fig. 2812 color, high-temperature and high-pressure dyeing machine are done by automation as shown in Fig. 29. There are base-outlet type and side-outlet type and its size is various $(610 \sim 1,220)$. The operation is done by the combination of electro-magnetic tap driven by oil pressure or air pressure with the timer. When once it is set according to the condition of original liquid and separation, a continious operation is possible.

The same automatic and continuous centrifugal seperator having base outlet was exhibited by Miyashita Co.) 


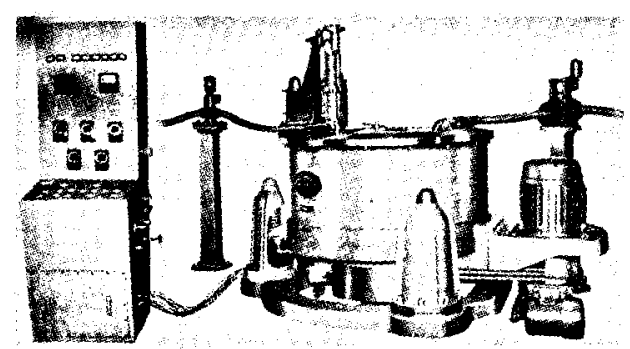

Fig. 29 Fully automatic centrifugal separator

(24) Suspended Type Centrifugal Seperator with Directly Connected Motor (Tanaka Machinery Co., Ltd.)

This is not as light like the one by Tanabe Iron Works Ltd. It is of fixed type and the transmission part is directly connected with the motor and suspended. The motor has been designed specially, and the speed changer is the patent of this company. It is attached with cooling instrument for brake drum and mechanical inter locking instrument. Fig. 31 shows its operation.

\section{(25) Ultra Filter (Miura Kagaku Sochi Co., Ltd.)}

It is a difficult thing to filter micro particles by the ordinary filter cloths but this machine can filter even colitis germs ( 0.1 micro) by using diatom earth as assistant. The use of diatom earth is not a new thing but the metal leaf of stainless steel for the filtering net is a new thing to be noted. This is shown in Fig. 32. Pre-coating column, pump have been included in the filtering column, where as the operation of them can be done by bulb. Cakes can be washed away by shower. There are various sizes and forms and its use is wide. The outlook is shown in Fig. 33, where it is connected in parallel.

\section{(26) Endless Cloth Belt Filtering Machine (Daiki Rubber Industrial Co., Ltd.)}

This type of filtering machine is not different to the vacuum sucktion type except that it has its own mechanism of eliminating cakes. As it has been shown in Fig. 34, by the roller No. 2. The cake can be cleaned from the back side by any suitable cleansing liquid. The cleaned filter can be returned after one revolution. It is suitable for the filtration of $\mathrm{CaSO}_{4} .2 \mathrm{H}_{2} \mathrm{O}$.

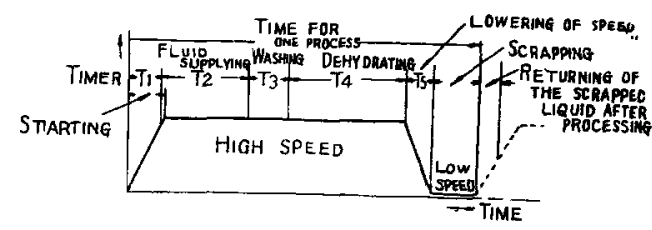

Fig. 30

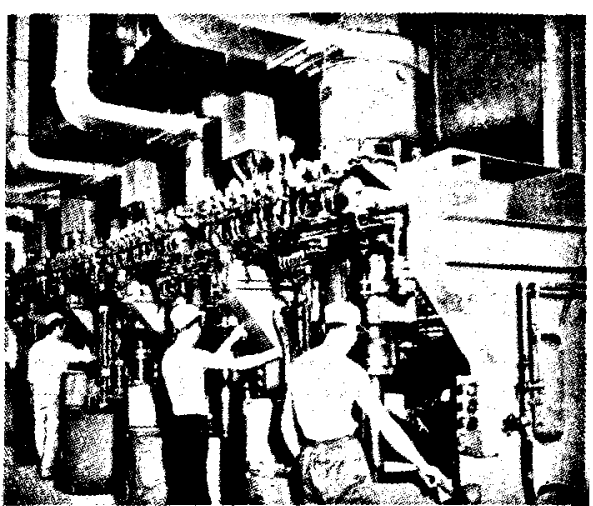

Fig. 31 Suspended type centrifugal seperator with directly connected motor

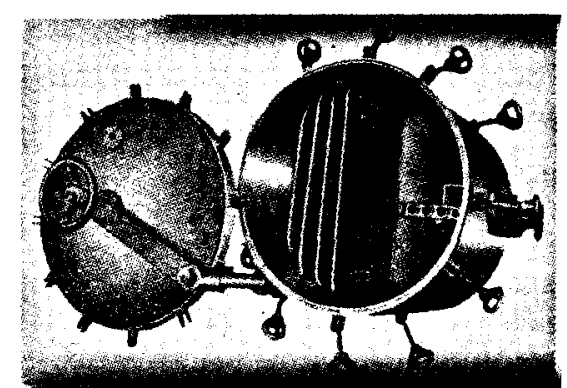

Fig. 32

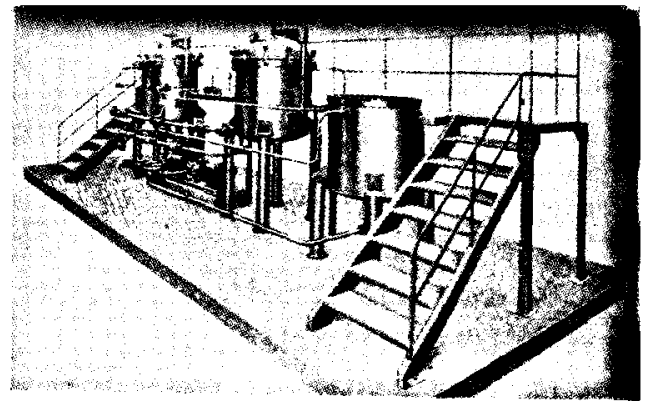

Fig. 33 


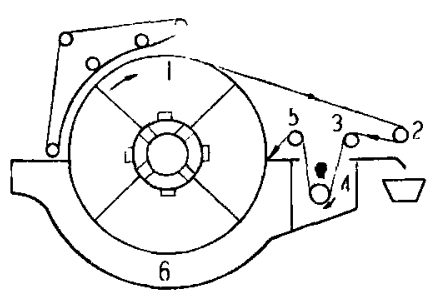
1. Drum
2. Eliminating roller
4. Washing wechanism
3. Roller
5. Roller
6. Liquids bath

Fig. 34

\section{(27) IDI-type Fine Winding Machine (Ichikin Kogyosha Co., Ltd.)}

When the winding diameter in Jigger and Tenter increases, the number of revolution of this machine is decreased and the rotating power is increased comparatively. This helps to stabilize the tension of the fabric. The differential gear adjusted in this machine plays the main role. The structure is simple and the body is light.
(28)

\section{Ebara-Inflico Cyculator (Ebara Seisakusho Ltd.)}

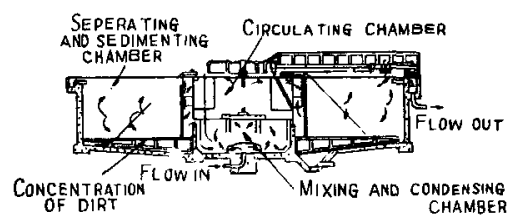

Fig 35 Sectional view

It is used for textile, paper, and pulp industry. The original liquid is mixed with chemicals and circulated in the direction shown in Fig. 35. The mixed liquid flows to the sedimentation chamber. The particles of sediments at the base flows back to the mixing and concentration chamber in a fixed amount. The concentrated sediments are accumu. lated in the dirt chamber. The washed water is re-feeded to the amount flowed in.
7. Sewing and Other Types of Finishing Machine

\section{(29) Tsugami Kilting Machine (Tsugami Seisaku- sho Co., Ltd.)}

The machine is shown in Fig. 36.

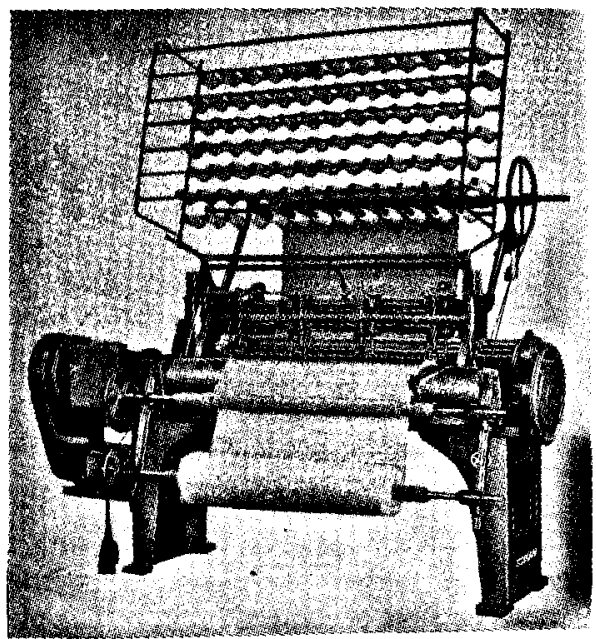

Fig. 36 Tsugami's kilting machine (Tsugami seisakusho Ltd.)

\section{Textile Testing Machine}

(30) Microscope and Projector (Hitachi Ltd. Asahi Sokki Co., Nihon Electric Co., Ltd., Union Optical Co.)

HS-6 type electron microscope was shown by Hitachi Seisakusho Ltd. The exciting current is not necessary due to the use of permanent magnet and the stability is better. The handling of it is simple without much damage. The changing of lenses having $10,20,50$ and 100 times magnifying power for the universal projector by Asahi Sokki Co., can be done instantly by turret system. The projecting plane is inclined at 260 degree to make the observation easier.

Infra-aed Microscope (Nihon Electric Co.)

It can be used for observing various samples in a range of wave length between $0.35 \sim 1.2$ microns. This can also be used in the range of visible wavelength between 0.4 to 0.76 microns. The light source is of passing and reflecting type. L-388 type image tube has been used for changing infrared ray image into visible one. This can be taken by $35 \mathrm{~mm}$ camera. Originally, this was manufactured for semi-conductor but it is possible to use for fiber. The magnifying 
power is $50 \sim 1500$ times.

HUM type vacuum high-temperature microscope was exhibited by Union Optical Co. The sensitivity of the light source can be adjusted and the highest temperature inside the oven is $1500^{\circ} \mathrm{C}$. The minimum degree of vacuum is $10^{5} \mathrm{~mm} \mathrm{Hg}$, and the time for making vacuum is 8 minutes. It can be taken by camera $(8 \mathrm{~mm}, 16 \mathrm{~mm})$ and the image is visible.

\section{(31) Thickness Measuring Meter by $\beta$ Ray (WD- 626 Type, Iwasaki Tsushinki $\mathrm{Co}$.)}

$\beta$ ray is passed on one side of the measuring sample and the other side of it is attached with the ionization chamber. $\beta$ ray ionizes the gaseous molecules in the box and the ionization current flows between the two poles. The fundamental equation for $\beta$ ray is $I=I_{0} e^{-\mu m x}$ where $x$ is thickness of the sample $\left(\mathrm{g} / \mathrm{m}^{2}\right)$. It is a problem whether it can be used in the textile industry as there is limit in the source. It is now being used for the paper industry. This instrument is equipped with double ionization system which corrects the temperature effect, as the ionization current varies with temperature. Fig. 37 shows the double ionization type (WD-626 B-type)

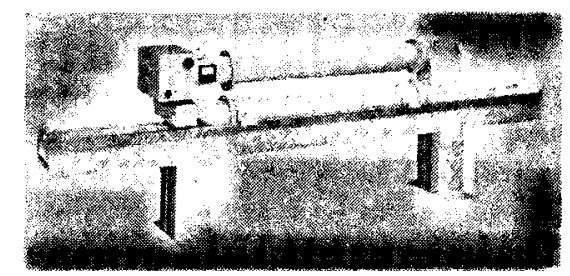

Fig. 37 Thickness measuring meter by $\beta$ ray

\section{(32) Durability Tester (Toyo Rika Kogyo Co.)}

Xenon lamp which has the same distribution of energy as that of solar ray has been used in Xenon Weather-O-Meter of Toyo Rika Kogyo Co. The number of samples can be tested at one time is 20 . The temperature can be varied between $0-80^{\circ} \mathrm{C}$, and the humidity can be controlled by hair hygrometer.

\section{(33) Dew Point Hygrometer (Sumitomo Electric Co., Ltd.)}

The metal plate of this instrument is cooled by electronic element and the cloud grown on the plate is taken by photo transistor. The temperature of the metal plate is kept constant by controlling the current flowing through the panel and is recorded by thermistor.

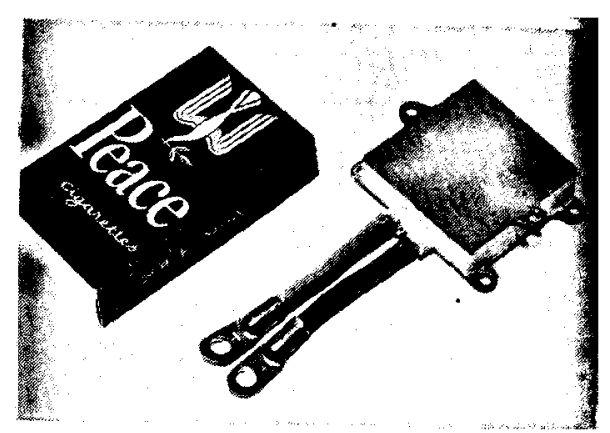

Fig. 38 Electronic cooling panel

\section{(34) Tensile Tester(Toyo Seiki Seisakusho Co., Ltd.)}

Digitron and T-Stro-Graph of the same company are not different in mechanism. Schopper tensile tester can be replaced by Digitron. This does not record stress-strain curve but can be read through digital tube. T-Stro-Graph is almost same to Instron tensile tester and it is attached with heating oven and cooling instrument. Cooling is done by dry ice and trichloro ethylene. The temperature can be varied from -50 to $150^{\circ} \mathrm{C}$.

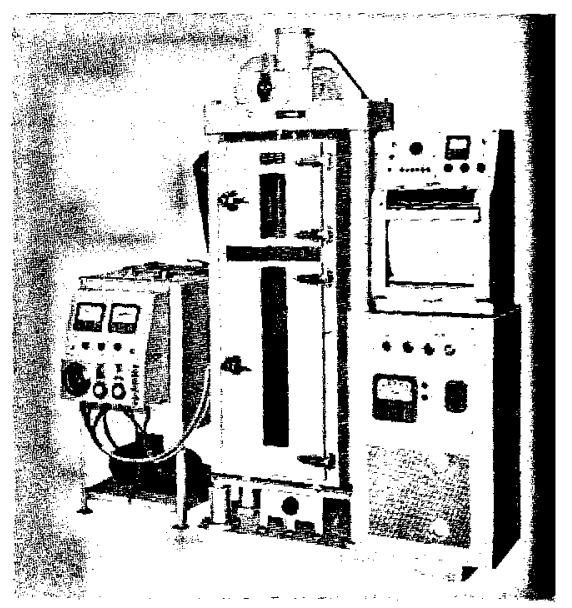

Fig. 39 T-stro-graph

(35) Color Difference Meter (Nihon Colour Machine Co., Ltd.)

CM-20 type color meter is attached with Xenon stabilizer which makes the spectrum of the light source same as that of natural light. The measurement of the flourescent light is also possible. The colour difference can be calculated by $M_{2}$ meter. 


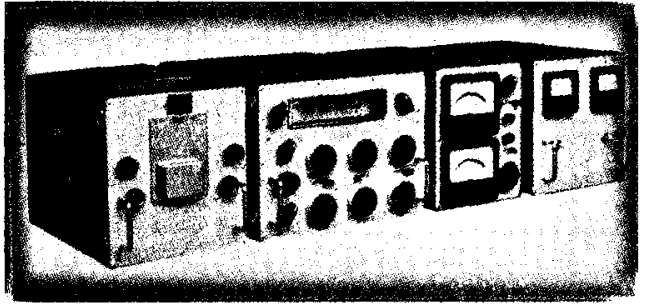

Fig. 40 Color difference meter (CM-20 type)

\section{(36) Calender Gauge (Densoku Kogyo Co., Ltd.)}

Fig. 41 shows the structure of calender gauge for measuring the thickness of non-magnetic body (such as rubber, plastic film, and lamination) during manufacture. The measuring limit is $0 \sim 3 \mathrm{~mm}$ and $0 \sim 0.5 \mathrm{~mm}$. The error is between $1 \%$ and the measuring pressure is $200 \sim 1000 \mathrm{~g}$.

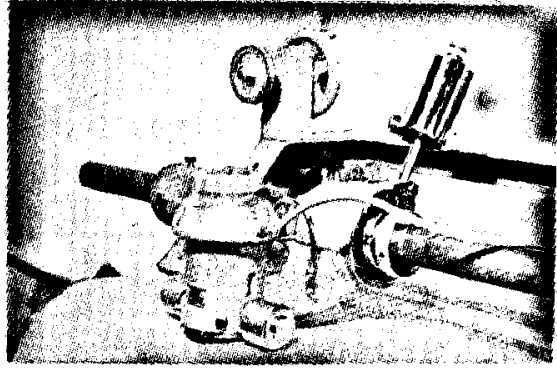

Fig. 41 Calender gauge

(37) Measurement of Relative Humidity of the Rotating Roller (Electric Instrument Keiki Co., Ltd.)

It is attached with index meter, adjustor, recorder etc. The heat evolved from the surface of the measuring body is dragged by the motor fan to the interior of the instrument, where it passes through the humidity sensitive resistor which records the humidity. 